\title{
Effect of combined treatment with rosuvastatin and protein kinase $C \beta 2$ inhibitor on angiogenesis following myocardial infarction in diabetic rats
}

\author{
DONG HUANG $^{1 *}$, FA-BIN WANG ${ }^{2 *}$, MING GUO $^{2}$, SHUAI LI $^{1}$, \\ MEI-LING YAN ${ }^{1}$, TAO YU ${ }^{1}$, MENG WEI ${ }^{1}$ and JING-BO LI ${ }^{1}$ \\ ${ }^{1}$ Division of Cardiology, The Sixth People's Hospital Affiliated to Shanghai Jiaotong University, \\ Shanghai Jiaotong University School of Medicine, State Key Discipline Division, Shanghai 200233; \\ ${ }^{2}$ Division of Cardiology, Tengzhou Central People's Hospital, Tengzhou, Shandong 277500, P.R. China
}

Received September 6, 2014; Accepted December 12, 2014

DOI: 10.3892/ijmm.2014.2043

\begin{abstract}
The aim of the present study was to investigate the effects of combined treatment with rosuvastatin and LY333531, a selective protein kinase $\mathrm{C}$ (PKC) $\beta 2$ inhibitor, on angiogenesis under hyperglycemic conditions. Human umbilical vein endothelial cells (HUVECs) cultured in medium containing a normal or high concentration of glucose $(33.3 \mathrm{mmol} / \mathrm{l})$ were treated with rosuvastatin $(0.1 \mu \mathrm{mol} / \mathrm{l})$ alone or in combination with LY333531 (10 nmol/l). HUVEC migration and tube formation were assessed. Furthermore, rats with streptozotocin-induced diabetes were randomly divided into groups and treated with either rosuvastatin alone $(5 \mathrm{mg} / \mathrm{kg} /$ day) or in combination with LY333531 (10 mg/kg/day) for 4 weeks following the induction of myocardial infarction (MI). Echocardiographic patterns, the extent of myocardial fibrosis, capillary density in myocardial tissue, the phosphorylation of Akt and endothelial nitric oxide synthase (eNOS), as well as the expression levels of vascular endothelial growth factor (VEGF) and hypoxia-inducible factor $1-\alpha(\mathrm{HIF}-1 \alpha)$ were assessed. The results from the in vitro experiment revealed that the tube-forming and migration ability of the HUVECs exposed to high-glucose medium was significantly improved in the group treated with the combination of rosuvastatin and LY333531. In vivo, the combination of rosuvastatin and LY333531 significantly improved left ventric-
\end{abstract}

Correspondence to: Professor Meng Wei or Professor Jing-Bo Li, Division of Cardiology, The Sixth People's Hospital Affiliated to Shanghai Jiaotong University, Shanghai Jiaotong University School of Medicine, 600 Yishan Road, Shanghai 200233, P.R. China

E-mail: mrweei@medmail.com.cn

E-mail: ljbsjtu6h@hotmail.com

${ }^{*}$ Contributed equally

Key words: rosuvastatin, protein kinase $\mathrm{C} \beta 2$, myocardial infarction, angiogenesis, diabetes ular function, reduced the extent of myocardial fibrosis and increased myocardial capillary density compared to treatment with rosuvastatin alone. In addition, the expression levels of VEGF, and Akt and eNOS phosphorylation were significantly higher in the group exposed to the combination treatment than in the group treated with rosuvastatin alone. The results of the present study indicate that, compared to treatment with rosuvastatin alone, combined treatment with rosuvastatin and LY333531 promotes a greater level of angiogenesis in diabetic rats with MI. This effect is likely mediated through the upregulation of the VEGF-dependent Akt/eNOS signaling pathway.

\section{Introduction}

Cardiovascular disease, a leading cause of mortality worldwide, commonly presents as myocardial infarction (MI) (1). The ischemia-induced formation of coronary collateral circulation serves as a compensatory mechanism that provides blood perfusion to ischemic myocardial tissue, thus playing a significant role in the prognosis of MI (2). Coronary artery disease, including MI, is also a common complication contributing to morbidity and mortality in diabetic patients (3). In diabetes, angiogenesis is impaired (4), which may underlie the poor prognosis of diabetic patients with cardiovascular complications (5). Therefore, therapeutic angiogenesis has been proposed as an exciting strategy to enhance perfusion to the ischemic myocardium of diabetic patients with MI.

Vascular endothelial growth factor (VEGF) plays a pivotal role in blood vessel formation during the developmental stage and in the regulation of hypoxia-induced angiogenesis (6). VEGF expression is regulated by hypoxia, oxidative stress and nitric oxide (NO), and elevated VEGF levels have been observed in the retina and glomeruli of diabetic rats $(7,8)$. However, the levels of both VEGF and its receptors are decreased in myocardial tissue in diabetic animals (9). This preclinical observation suggests a potential molecular explanation for inadequate collateral vascular formation in response to myocardial ischemia in diabetic patients.

Protein kinase C (PKC) is a family of cytoplasmic serine/threonine kinases with a wide variety of functions in 
intracellular signal transduction (10). The activation of PKC is associated with vascular complications under diabetic conditions (11). The $\beta$ isoforms of PKC (PKC $\beta 1$ and $\mathrm{PKC} \beta 2)$ are expressed in cardiovascular tissue and can be activated by free fatty acids and under hyperglycemic conditions $(12,13)$. In diabetic rats, the activation of PKC $\beta$ is elevated in vascular tissue and is involved in the dysfunction of the cardiac microvascular barrier (14). Furthermore, in endothelial cells, PKC $\beta$ has been shown to inhibit the activation of Akt by insulin or VEGF, and to interrupt the regulation of Akt-dependent endothelial NO synthase (eNOS) by insulin in obesity-associated insulin resistance (15). In a previous study, a PKC $\beta$-selective inhibitor, LY333531, was shown to prevent diabetic vascular complications both in vitro and in vivo (16). However, the effects of the inhibition of PKC $\beta$ on angiogenesis in the ischemic myocardium under diabetic conditions remain unclear.

Statins are inhibitors of the 3-hydroxy-3-methylglutaryl coenzyme A (HMG-CoA) reductase, the rate-limiting enzyme in cholesterol biosynthesis. This class of drugs can also induce lipid-independent benefits, including the improvement of endothelial function, the inhibition of inflammation, the facilitation of angiogenesis and, consequently, the reduction of the myocardial or cerebral infarct size (17). The promoting effect of statins on angiogenesis is likely mediated through the VEGF-dependent Akt/eNOS pathway. Accordingly, statins have been shown to promote angiogenesis in response to ischemia, which is mediated by $\operatorname{VEGF}(18,19)$. In the present study, we therefore aimed to investigate the effects of combined treatment with rosuvastatin and LY333531 on angiogenesis in myocardial tissue in diabetic rats with MI.

\section{Materials and methods}

Cell culture and treatments. Human umbilical vein endothelial cells (HUVECs) were purchased from the American Type Culture Collection (ATCC, Manassas, VA, USA). The cells were cultured in Dulbecco's modified Eagle's medium (DMEM) supplemented with $10 \%$ bovine serum albumin (BSA; Gibco-Invitrogen Inc., Carlsbad, CA, USA), $0.05 \mathrm{mg} / \mathrm{ml}$ endothelial growth factor (Sigma, St. Louis, MO, USA), $0.1 \mathrm{mg} / \mathrm{ml}$ heparin, 100 units $/ \mathrm{ml}$ penicillin and $100 \mathrm{mg} / \mathrm{ml}$ streptomycin at $37^{\circ} \mathrm{C}$ under $5 \% \mathrm{CO}_{2}$. Cells between the second and fourth passages were used for the experiments. The cells were divided into 5 treatment groups as follows: i) cells cultured in normal glucose medium (N-G, $5.6 \mathrm{mmol} / \mathrm{l})$; ii) cells cultured in high glucose medium (H-G, $33.3 \mathrm{mmol} / \mathrm{l})$; iii) cells cultured in high glucose medium and treated with rosuvastatin (RSV, $0.1 \mu \mathrm{mol} / \mathrm{l})$; iv) cells cultured in high glucose medium and treated with LY333531 (LY, $10 \mathrm{nmol} / \mathrm{l}$ ); and v) cells cultued in high glucose medium and treated with rosuvastatin plus LY333531 (RSV + LY, $0.1 \mu \mathrm{mol} / 1+10 \mathrm{nmol} / \mathrm{l})$. Rosuvastatin (obtained from AstraZeneca, Cheshire, UK) and LY333531 (from Eli Lilly \& Co., Indianapolis, IN, USA) were dissolved in dimethyl sulfoxide (DMSO, 0.2\%; Sigma).

Tube formation assay. The formation of tube-like structures by HUVECs on Matrigel (BD Biosciences, Oxford, UK) was assessed as previously described (20). The cells were seeded in gel-precoated wells and cultured in the absence or presence of either rosuvastatin alone or in combination with LY333531 in $\mathrm{H}-\mathrm{G}$ medium at $37^{\circ} \mathrm{C}$ for $24 \mathrm{~h}$ with $5 \% \mathrm{CO}_{2}$. Cells cultured in $\mathrm{N}-\mathrm{G}$ medium served as the controls. Tube formation was observed under a microscope (Olympus Corp., Tokyo, Japan). Images were analyzed using Image-Pro Plus software (Media Cybernetics, Inc., Rockville, MD, USA). The degree of tube formation was assessed by measuring the length of tubes in 3 random fields (magnification, x40) from each well. Tube formation under different treatment conditions was normalized to that under normal glucose conditions.

Migration assay. A wound healing assay was performed to measure the unidirectional migration of HUVECs, as previously described (21). The cells were seeded in a 6-well plate at a density of $5 \times 10^{4}$ cells/well, and incubated at $37^{\circ} \mathrm{C}$ with $5 \% \mathrm{CO}_{2}$ for $24 \mathrm{~h}$. After being washed with PBS twice, the cells were incubated in $\mathrm{N}-\mathrm{G}$ medium containing $1 \% \mathrm{FBS}$ for $12 \mathrm{~h}$. The wells were then scraped with a $200-\mu \mathrm{l}$ pipette tip to mechanically injure the cells. The wells were rinsed with PBS twice to remove cellular debris and dislodge the cells. After injury, the cells were cultured in the presence of the different reagents for $24 \mathrm{~h}$. Images were captured $24 \mathrm{~h}$ later using an Olympus microscope (Olympus Corp.) at x40 magnification. The migration ability of the cells was calculated using the following formula: $100 \%$ (width at $24 \mathrm{~h} /$ width at $0 \mathrm{~h}$ ) x100\%, as previously described (22).

Cell proliferation assay. Cell proliferation was assessed using the 3-(4,5-dimethylthiazol-2-yl)-2,5-diphenyltetrazolium bromide (MTT) assay (Sigma). The HUVECs were seeded into 96-well plates at a density of 2,000 cells/well and were incubated in N-G medium containing $1 \% \mathrm{FBS}$ at $37^{\circ} \mathrm{C}$ with $5 \% \mathrm{CO}_{2}$ for $24 \mathrm{~h}$. The cells were then transferred to fresh medium in the absence or presence of the different reagents. Each group had 3 replicate wells. Following incubation for different periods of time, $10 \mu \mathrm{l}$ of MTT were added to each well and the cells were further incubated at $37^{\circ} \mathrm{C}$ for $4 \mathrm{~h}$ with $5 \% \mathrm{CO}_{2}$. The supernatant fluid was then removed and $100 \mu 1$ of DMSO were added to each well. The absorbance (OD value) at a wavelength of $490 \mathrm{~nm}$ was measured using a microplate reader (Bio-Tek ELx808; BioTek Instruments, Inc., Winooski, VT, USA), as previously described (23).

Animal model. Male Sprague-Dawley (SD) rats, (weighing 200-250 g, from the Experimental Animal Center, Fudan University, Shanghai, China) were used in the experiment. All procedures were approved by the Animal Care and Use Committee of the Sixth People's Hospital affiliated to Shanghai Jiaotong University, Shanghai, China and were in compliance with the regulations of the Animal Welfare Act of the National Institutes of Health Guide for the Care and Use of Laboratory Animals. The animals were allowed to acclimatize for 2 weeks prior to the experiment.

After an overnight fast, the animals were administered streptozotocin [50 mg/kg, intraperitonealy (i.p.) daily] (Sigma) or the vehicle (citrate saline, i.p.) (14). One week after the streptozotocin injection, blood glucose levels were measured. Animals with a glucose level $\leq 300 \mathrm{mg} / \mathrm{dl}$ were excluded from the study.

After 1 week of the administration of the streptozotocin injection, the model of MI was created as described in our 
previous study (24). In brief, a total of 55 diabetic rats were anesthetized by an intraperitoneal injection of pentobarbital (50 $\mathrm{mg} / \mathrm{kg}$ ) and were ventilated using an animal respirator (DW-200; Alcott Biotech, Shanghai, China) with room air following endotracheal intubation. The electrical activity of the mouse hearts was continuously monitored during the experiment using an lead II electrocardiogram (ECG). Heart exposure was performed by a thoracotomy at the left fourth intercostal space. The left anterior descending coronary artery (LAD) was ligated using a 6-0 prolene suture, 1-2 mm below the tip of the left atrial appendage. LAD ligation was confirmed by ST segment elevation. Another 10 diabetic rats, as a control group, underwent thoracotomy and incision of the pericardial sac, but not LAD ligation.

Animal protocols. After establishing the animal model, all surviving animals were randomly assigned into the following groups: i) the MI group: rats with MI were treated with the vehicle (normal saline orally) for 4 weeks starting 1 day after LAD ligation; ii) the RSV group: rats with MI were administered rosuvastatin orally ( $5 \mathrm{mg} / \mathrm{kg}$ daily); iii) the LY333531 group: rats with MI were administered LY333531 orally (10 mg/kg daily); iv) the RSV + LY333531 group: rats with MI were orally administered rosuvastatin $(5 \mathrm{mg} / \mathrm{kg}$ daily) plus LY333531 (10 mg/kg daily); and v) another sham-operated and non-diabetic group of animals was administered the vehicle (normal saline). All surviving animals were sacrificed on day 28. Before the rats were sacrificed, an echocardiographic examination was performed to evaluate the remodeling process.

After the rats were euthanized, whole blood was collected from the inferior vena cava and the hearts were harvested. One cross-section of left ventricular (LV) myocardial tissue at the level of the papillary muscles, approximately $2 \mathrm{~mm}$, was collected and fixed in $10 \%$ formalin followed by embedding in paraffin for histological examination. The remaining LV tissue was quickly separated into the peri-infarct zone and remote zone, and the tissue was snap-frozen and mechanically homogenized in liquid nitrogen, and resuspended in lysis buffer to which phosphatase was added. Subsequently, the tissue was further lysed in a tightly fitting cylinder homogenizer. The sample was then stored at $-80^{\circ} \mathrm{C}$ for later analysis.

Echocardiography. An echocardiographic examination was performed on day 28 following the induction of MI using a standard Acuson Sequoia 512 ultrasound system equipped with a $15-\mathrm{MHz}$ probe (Siemens, Erlangen, Germany). Both two-dimensional and M-mode echocardiography were performed. The LV end-diastolic diameter (LVEDD) and the LV end-systolic diameter (LVESD) were measured. In addition, the LV ejection fraction (LVEF) and fractional shortening (FS) were calculated, as previously described (25). All measurements from 5 consecutive cardiac cycles were averaged and analyzed by a single observer blinded to the treatment protocol.

Histological analysis. Under deep anesthesia with pentobarbital sodium (60 mg/kg, i.p.), the heart of a rat was excised and cut into transverse slices (2-mm-thick), which were then fixed in $10 \%$ formalin solution for $24 \mathrm{~h}$. The samples were then embedded in paraffin and sectioned at $4 \mu \mathrm{m}$. Immunohistochemical analysis was performed as previ- ously described (26). In brief, deparaffinized tissue sections (4- $\mu \mathrm{m}$-thick) were blocked by $10 \%$ goat serum for $30 \mathrm{~min}$ at $37^{\circ} \mathrm{C}$. Rabbit polyclonal antibodies against CD31 (ab28364; capillary density maker; diluted at 1:500) and VEGF (ab46154; diluted at 1:250; Abcam, Cambridge, MA, USA) were employed as primary antibodies. Following overnight incubation with the primary antibodies at $4^{\circ} \mathrm{C}$, the sections were treated with goat polyclonal secondary antibody to rabbit IgG (goat anti-rabbit IgG H\&L, ab97047; diluted at 1:1,000; Abcam). All sections were counterstained with hematoxylin. Immunoreactivity for CD31 and VEGF was measured using the Image-Pro Plus 4.0 analysis system (Media Cybernetics, Inc., Bethesda, MD, USA). Capillaries were identified by positive staining for CD31. The results were expressed as an average amount of capillaries per x10 field, as previously described (27).

Tissue staining with Masson's trichrome stain was performed in the border area to evaluate collagen deposition. Sirius Red Staining (a collagen-specific dye) was also used to clearly discriminate between the cardiomyocytes and collagen. Collagen volume fraction was analyzed using ImageJ software and expressed as the average percentage of collagen staining of 20 randomized high power fields.

Enzyme-linked immunosorbent assay (ELISA). The plasma levels of transforming growth factor (TGF)- $\beta 1$, VEGF and hypoxia-inducible factor (HIF)-1 $\alpha$ were determined using solid-phase sandwich ELISA kits (USCN Life Science Inc., Wuhan, China), according to the manufacturer's instructions.

Reverse transcription-quantitative polymerase chain reaction (RT-qPCR). Total RNA was prepared from $150 \mathrm{mg} \mathrm{LV}$ free wall using TRIzol reagent (Invitrogen, Life Technologies), followed by chloroform extraction and isopropanol precipitation. Genomic DNA was eliminated by incubating with DNase I $\left(0.1 \mu \mathrm{l}^{-1}, 37^{\circ} \mathrm{C}\right)$ for $30 \mathrm{~min}$ followed by acid phenol-chloroform extraction. RNA was quantified by spectrophotometric absorbance at $260 \mathrm{~nm}$ (A260), with its purity confirmed by measuring the $A_{260} / A_{280}$ ratio. The integrity of RNA was evaluated by ethidium bromide staining on a denaturing agarose gel. Total RNA $(2 \mu \mathrm{g})$ was then reverse transcribed using oligo(dT) primer and SuperScript II RT (Invitrogen-Life Technologies, Carlsbad, CA, USA).

Quantitative (real-time) PCR was performed as described in a previous study of ours (28) starting with $12.5 \mathrm{ng}$ cDNA and both sense and antisense primers at a concentration of $900 \mathrm{nM}$ (Invitrogen-Life Technologies) in a final volume of $25 \mu 1$, using SYBR-Green Master Mix (Applied Biosystems, Denver, CO, USA). Fluorescence was monitored and analyzed using a GeneAmp 7000 detection system instrument (Applied Biosystems). The PCR reactions were performed 42 times by a three-step cycle procedure (denaturation $95^{\circ} \mathrm{C}, 15 \mathrm{sec}$; annealing $60^{\circ} \mathrm{C}, 30 \mathrm{sec}$; extension $72^{\circ} \mathrm{C}, 30 \mathrm{sec}$ ) following an initial stage $\left(95^{\circ} \mathrm{C}, 10 \mathrm{~min}\right)$. A $\Delta \mathrm{Ct}$ value was obtained to quantify the $\mathrm{mRNA}$ levels and was normalized to an endogenous control (GAPDH mRNA) for each sample. A relative quantification $\Delta \Delta \mathrm{Ct}$ method was used for the comparison between groups. Oligonucleotide primers were designed using Primer Express software (Applied Biosystems). The primers used in this study were as follows: VEGF forward, 5'-CGAGACGCA 


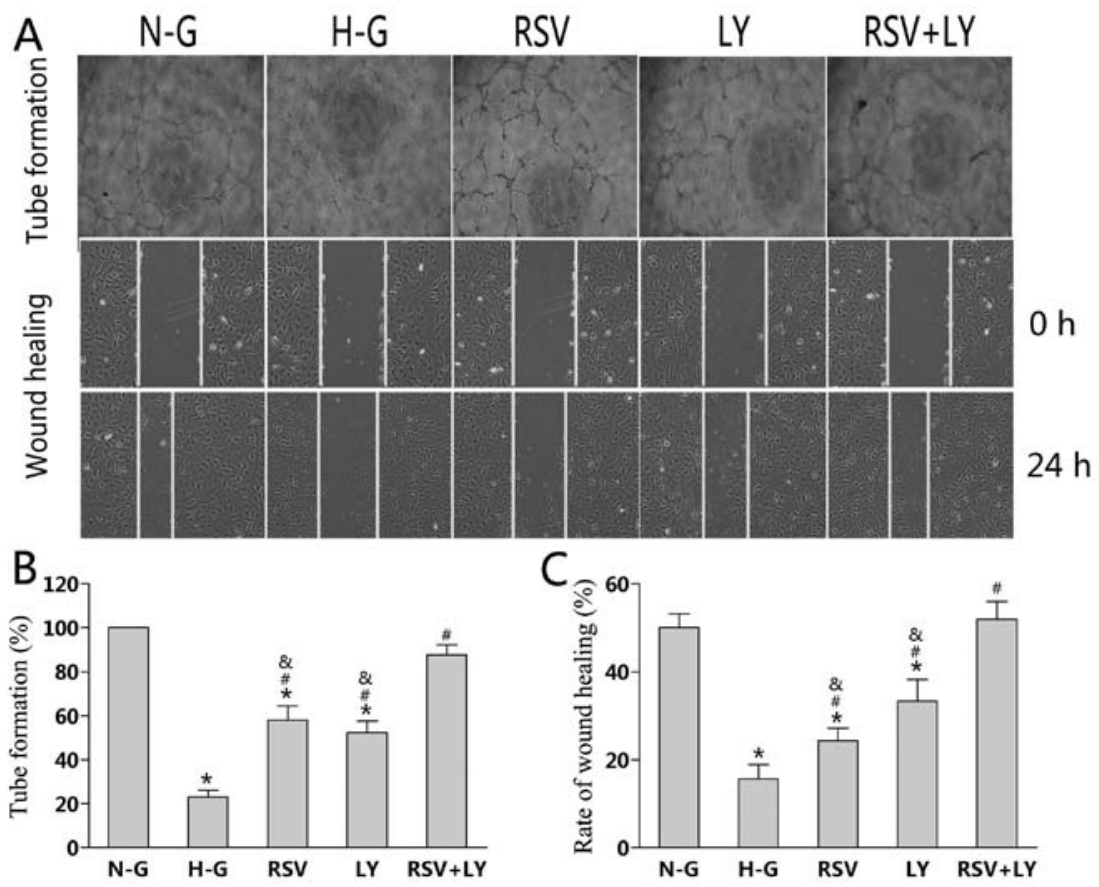

Figure 1. Matrigel assay was used to evaluate tube formation after $24 \mathrm{~h}$ under different treatment conditions. (A, upper panel) Tube formation was visualized using an Olympus microscope; representative fields (magnification, $\mathrm{x} 40$ ) are shown. (A, middle and lower panel) Wound healing assay was used to evaluate the migration of human umbilical vein endothelial cells (HUVECs) under different treatment conditions. Representative images were captured at time zero (A, middle panel) and at $24 \mathrm{~h}$ (A, lower panel) using an Olympus microscope at x40 magnificaiotn. Quantification of HUVEC tube formation (B) and wound healing (C). Data are expressed as the means $\pm \mathrm{SD}$. ${ }^{\mathrm{P}}<0.05$ vs. N-G group; ${ }^{\mathrm{P}} \mathrm{P}<0.05$ vs. H-G group; ${ }^{\mathrm{P}} \mathrm{P}<0.05 \mathrm{vs}$. RSV $+\mathrm{LY}$ group. N-G, normal glucose; H-G, high glucose; RSV, rosuvastatin; LY, LY333531.

GCGACAAGGCA-3' and reverse, 5'-ACCTTCTCCAAA CCGTTGGCA-3'; HIF-1 $\alpha$ forward, 5'-GCCCAGTGAGAAA GGGGAAA-3' and reverse, 5'-CGGCTGGTTACTGCT GGTAT-3'; and GAPDH sense, 5'-TGATGGGTGTGAA CCACGAG-3' and antisense, 3'-CCCTTCCACGATGCC AAAGT-5'.

Western blot analysis. The infarcted heart tissue from the LV free wall or the non-infarcted heart tissue at the same site was used for western blot analysis. Equal amounts of protein $(30 \mu \mathrm{g})$ were separated on a $8-12 \%$ Tris-glycine gel (Novex; Invitrogen-Life Technologies) and transferred onto polyvinylidene difluoride (PVDF) membranes (Roche Applied Science, Penzberg, Germany). After blocking with $5 \%$ skim milk, the membranes were incubated with primary antibodies against phospho-Akt Ser473 (sc-7985-R; 1:1,000 dilution; Santa Cruz Biotechnology, Santa Cruz, CA, USA) and phospho-eNOS Thr1117 (sc-21871-R; 1:500 dilution; Santa Cruz Biotechnology) overnight at $4^{\circ} \mathrm{C}$, followed by incubation with horseradish peroxidase (HRP)-conjugated secondary antibody. Immunoreactions were visualized using an ECL detection kit (Amersham Biosciences, Piscataway, NJ, USA). All protein expression levels were adjusted against GAPDH intensity (cat. no. 2118; Cell Signaling Technology, Danvers, MA, USA). Bands were quantified by densitometry using Image J software (version 1.41; National Institutes of Health, Bethesda, MD, USA).

Statistical analysis. Data are expressed as the means \pm SD. All data analyses were performed using SPSS 13.0 statistical software (SPSS, Inc., Chicago, IL. USA). One-way ANOVA followed by the Student-Newman-Keuls test was used to compare the effects of the different treatments on various parameters. A P-value $<0.05$ was considered to indicate a statistically significant difference.

\section{Results}

Effect of combination treatment with rosuvastatin and LY333531 on tube formation and migration of HUVECs. Ten hours after plating, the HUVECs cultured in N-G and H-G medium treated with rosuvastatin + LY333531 had an elongated shape and had invaded the collagen gel to form tubes. By contrast, the HUVECs cultured in H-G medium remained in individual clusters. Twenty-four hours after plating, the HUVECs cultured in N-G medium formed a more extensive tube network than the HUVECs cultured in $\mathrm{H}-\mathrm{G}$ medium $(\mathrm{P}<0.01$; Fig. 1A). Single treatment with either rosuvastatin or LY333531 significantly promoted tube formation by HUVECs compared to the H-G goup ( $\mathrm{P}<0.05 ; \mathrm{Fig}$. 1B). The tube-forming ability of the HUVECs was more enhanced following treatment with rosuvastatin + LY333531 than with treatment with either agent alone ( $\mathrm{P}<0.05$; Fig. 1B).

Wound healing was assessed following culture for $24 \mathrm{~h}$ under different conditions (Fig. 1A). Compared to the $\mathrm{N}-\mathrm{G}$ group, culture in $\mathrm{H}-\mathrm{G}$ medium inhibited the wound healing ability of the HUVECs $(\mathrm{P}<0.05$; Fig. 1C). Treatment with rosuvastatin or LY333531 alone increased the wound healing ability of the HUVECs by 10 and $18 \%$, respectively, as compared to the $\mathrm{H}-\mathrm{G}$ group $(\mathrm{P}<0.05)$. Treatment with 
A
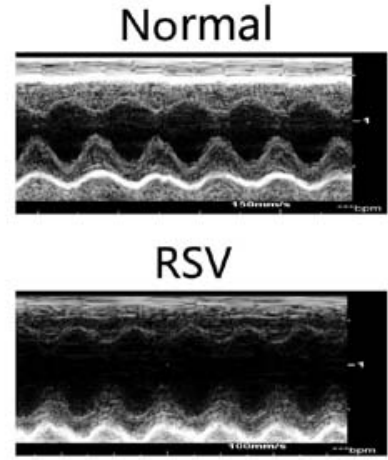

Diabetic sham

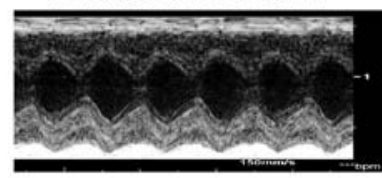

LY

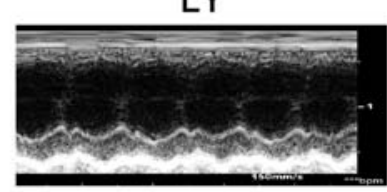

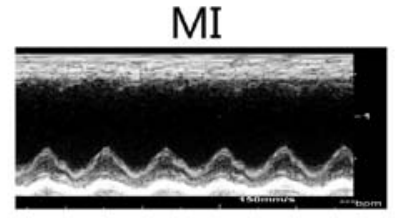

$\mathrm{RSV}+\mathrm{LY}$
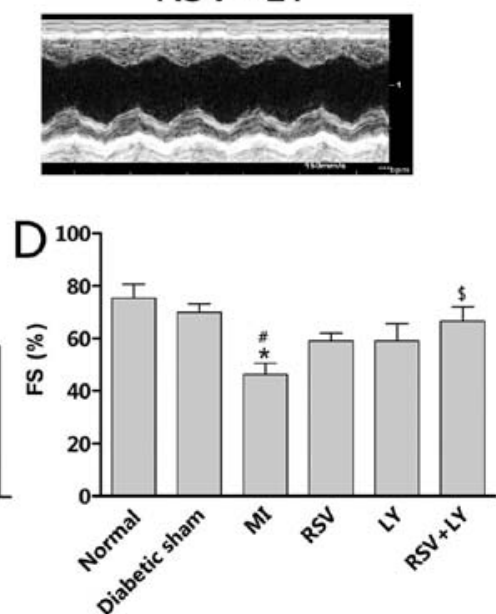

Figure 2. (A) Representative M-mode echocardiograms obtained from the normal (non-diabetic), diabetic sham-operated, RSV, LY and RSV + LY group rats. Summary data of echocardiographic measurements in each group of animals: (B) LVESD, (C) LVEDD and (D) FS. Data are expressed as the means \pm SD; $n=8-10$ rats per group. ${ }^{*} \mathrm{P}<0.05$ vs. normal group; ${ }^{\prime} \mathrm{P}<0.05$ vs. diabetic sham-operated group; ${ }^{\$} \mathrm{P}<0.05$ vs. MI group. Sham, sham-operated; RSV, rosuvastatin; LY, LY333531; MI, myocardial infarction; LVESD, left ventricular end-systolic diameter; LVEDD, left ventricular end-diastolic diameter; FS, fractional shortening.

rosuvastatin + LY333531 further facilitated wound closure; an increase of $32 \%$ was observed (Fig. 1C) compared to the cells treated with either agent alone $(\mathrm{P}<0.05)$.

Effect of combination treatment with rosuvastatin and LY333531 on cardiac function in rats. Twenty-eight days following surgery, the rats with MI demonstrated LV dilation, a greater LVESD and LVEDD, and decreased FS in comparison to the sham-operated rats $(\mathrm{P}<0.05$; Fig. 2$)$. Treatment with rosuvastatin + LY333531 improved these parameters $(\mathrm{P}<0.05$; Fig. 2B-D), while treatment with either rosuvastatin or LY333531 alone numerically decreased LVESD and LVEDD althgouth no statistically significant differences were observed $(\mathrm{P}>0.05$; Fig. 2B and C).

Effect of combination treatment with rosuvastatin and LY333531 on myocardial fibrosis in rats. The rats with MI had increased serum TGF- $\beta 1$ levels and myocardial interstitial fibrosis compared to the diabetic rats in the sham-operated group ( $\mathrm{P}<0.05$; Fig. 3A and $\mathrm{B})$. Treatment with rosuvastatin, LY333531, or rosuvastatin + LY333531 for 28 days reduced serum TGF- $\beta 1$ levels $(\mathrm{P}<0.05$; Fig. $3 \mathrm{~A})$. Treatment with rosuvastatin + LY333531, as compared to treatment with either agent alone, induced a greater reduction in interstitial fibrosis $(\mathrm{P}<0.05$; Fig. 3B) as indicated by Masson's thrichrome staining (Fig. 3C) and Sirius Red Staining (Fig. 3D).

Effect of combination treatment with rosuvastatin and LY333531 on capillary density in rats. The induction of MI in the rats increased capillary density in the peri-infarct zone as compared to rats in the sham-operated group $(\mathrm{P}<0.05$; Fig. $4 \mathrm{~A}$ and B). Treatment with either rosuvastatin or LY333531 alone did not change the capillary density, while treatment with rosuvastatin + LY333531 increased capillary density $(\mathrm{P}<0.05$; Fig. 4B). The expression levels of VEGF were increased after the induction of MI $(\mathrm{P}<0.05$; Fig. $4 \mathrm{C}$ and D). Treatment with rosuvastatin alone or rosuvastatin + LY333531 further increased the VEGF expression levels ( $\mathrm{P}<0.05$; Fig. 4D).

Effect of combination treatment with rosuvastatin and LY333531 on VEGF and HIF-1 $\alpha$ expression in rats. The rats with MI had increased serum VEGF levels compared with the rats in the sham-operated group $(79 \pm 6.1$ vs. $31 \pm 4.9 \mathrm{pg} / \mathrm{ml}$; $\mathrm{P}<0.05$; Fig. 5A). Treatment with rosuvastatin alone $(132 \pm 7.0$ vs. $79 \pm 6.1 \mathrm{pg} / \mathrm{ml}, \mathrm{P}<0.05)$, but not with LY333531 alone $(108 \pm 8.7 \mathrm{pg} / \mathrm{ml})$, further elevated the serum VEGF levels. Treatment with rosuvastatin + LY333531 induced a significant increase in VEGF levels compared to treatment with rosuvastatin alone ( $167 \pm 7.2$ vs. $132 \pm 7.0 \mathrm{pg} / \mathrm{ml}, \mathrm{P}<0.05$; Fig. $5 \mathrm{~A})$. MI increased myocardial VEGF mRNA levels (Fig. 5C). Treatment with rosuvastatin alone $(1.53 \pm 0.10$-fold increase, $\mathrm{P}<0.05$; Fig. 5C) or in combination with LY333531 further elevated the VEGF mRNA levels (1.56 \pm 0.13 -fold increase, $\mathrm{P}<0.05$; Fig. $5 \mathrm{C})$ compared with the MI group. There were no significant differences observed between single and co-treatment groups.

MI did not significantly alter the myocardial VEGF protein levels (Fig. 5E). Treatment with either rosuvastatin or LY333531 alone did not affect the VEGF protein levels, while higher VEGF protein levels were observed following combination treatment $(\mathrm{P}<0.05$; Fig. 5E). 


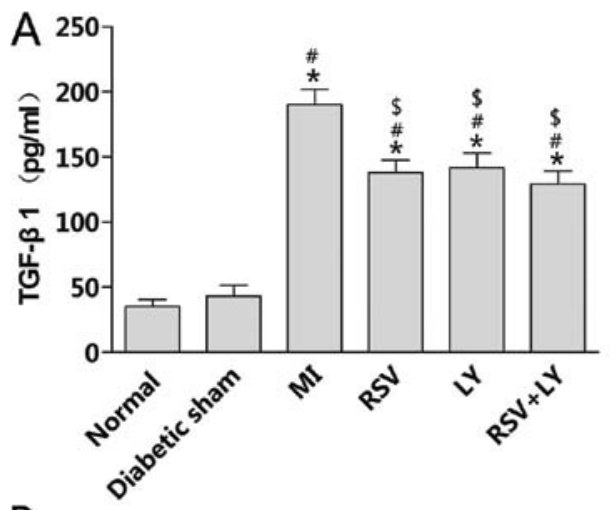

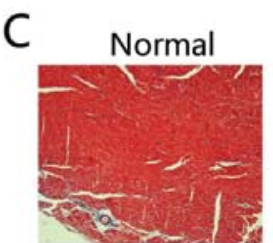

RSV
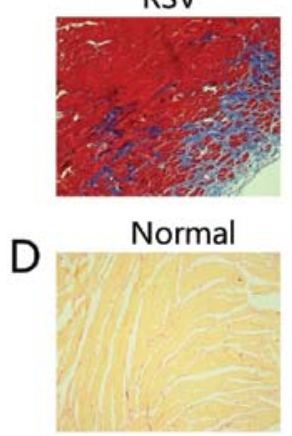

RSV

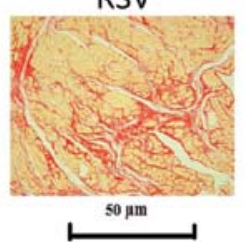

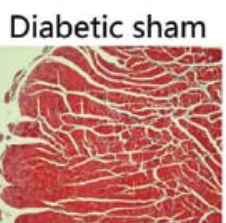

LY

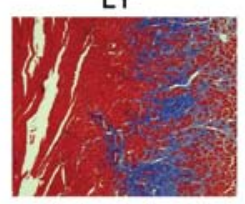

Diabetic sham

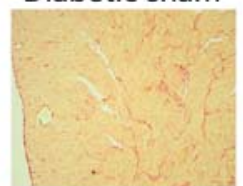

LY

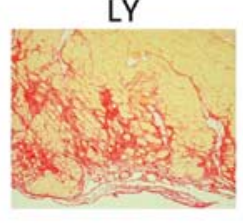

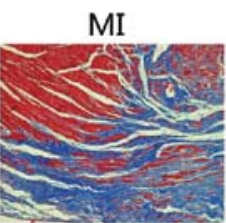

$\mathrm{RSV}+\mathrm{LY}$

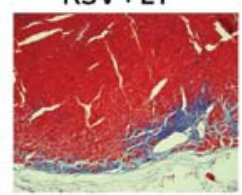

MI

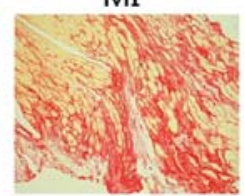

$\mathrm{RSV}+\mathrm{LY}$

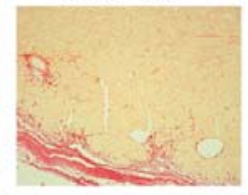

Figure 3. (A) Protein expression levels of TGF- $\beta 1$ in plasma were quantified by ELISA. (C) Representative sections of interstitial fibrosis (fibrotoc areas stained in blue, magnification x400; scale bar, $50 \mu \mathrm{m}$ ) obtained from each group and (B) respective quantifications are shown. (D) Sirius Red Staining was used to further confirm the pattern of interstitial fibrosis in each group with Masson's staining. Data are expressed as the means $\pm \mathrm{SD} ; \mathrm{n}=8-10$ rats per group. ${ }^{*} \mathrm{P}<0.05$ vs. normal group; ${ }^{\mathrm{P}} \mathrm{P}<0.05$ vs. diabetic sham group; ${ }^{\mathrm{S}} \mathrm{P}<0.05$ vs. MI group; ${ }^{\circledR} \mathrm{P}<0.05$ vs. RSV + LY group.TGF- $\beta 1$, transforming growth factor- $\beta 1 ; \mathrm{RSV}$, rosuvastatin; LY, LY333531; MI, myocardial infarction.

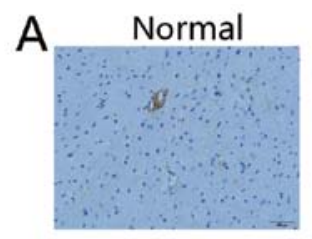

RSV

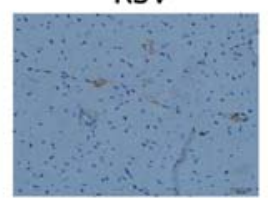

C

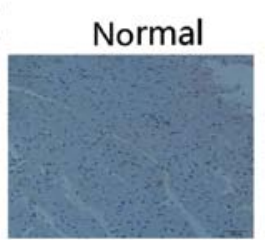

RSV

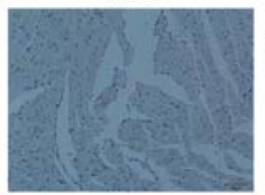

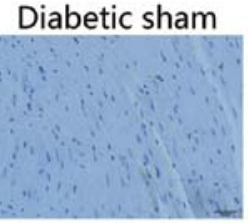

LY
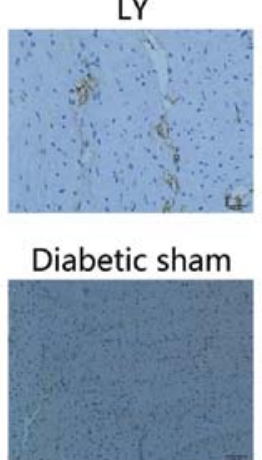

LY

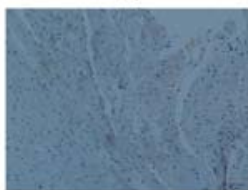

MI

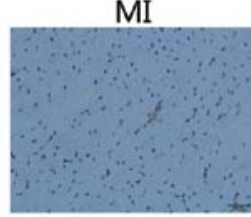

$R S V+L Y$

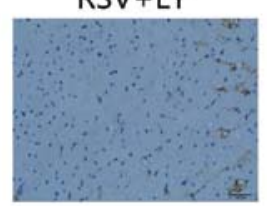

MI

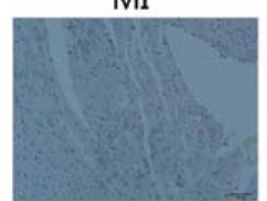

$\mathrm{RSV}+\mathrm{LY}$

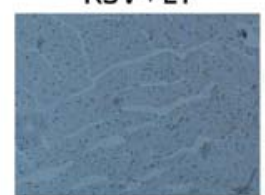

B
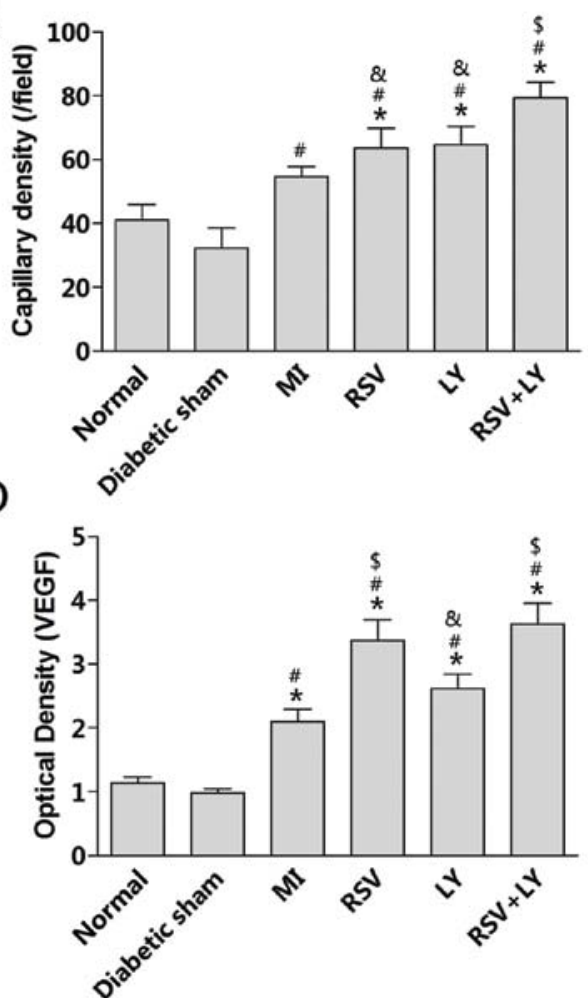

Figure 4. Representative slides of tissue from peri-infarct myocardium with (A) CD31 and (C) VEGF staining in each group. Scale bar, $100 \mu \mathrm{m}$. Quantification of (B) capillary density and (D) VEGF expression in each group is shown. Data are expressed as the means $\pm \mathrm{SD} ; \mathrm{n}=8-10$ rats per group. ${ }^{*} \mathrm{P}<0.05$ vs. normal group; ${ }^{~} \mathrm{P}<0.05$ vs. diabetic sham group; ${ }^{\$} \mathrm{P}<0.05$ vs. MI group; ${ }^{\circledR} \mathrm{P}<0.05$ vs. RSV + LY group. VEGF, vascular endothelial growth factor; RSV, rosuvastatin; LY, LY333531; MI, myocardial infarction. 

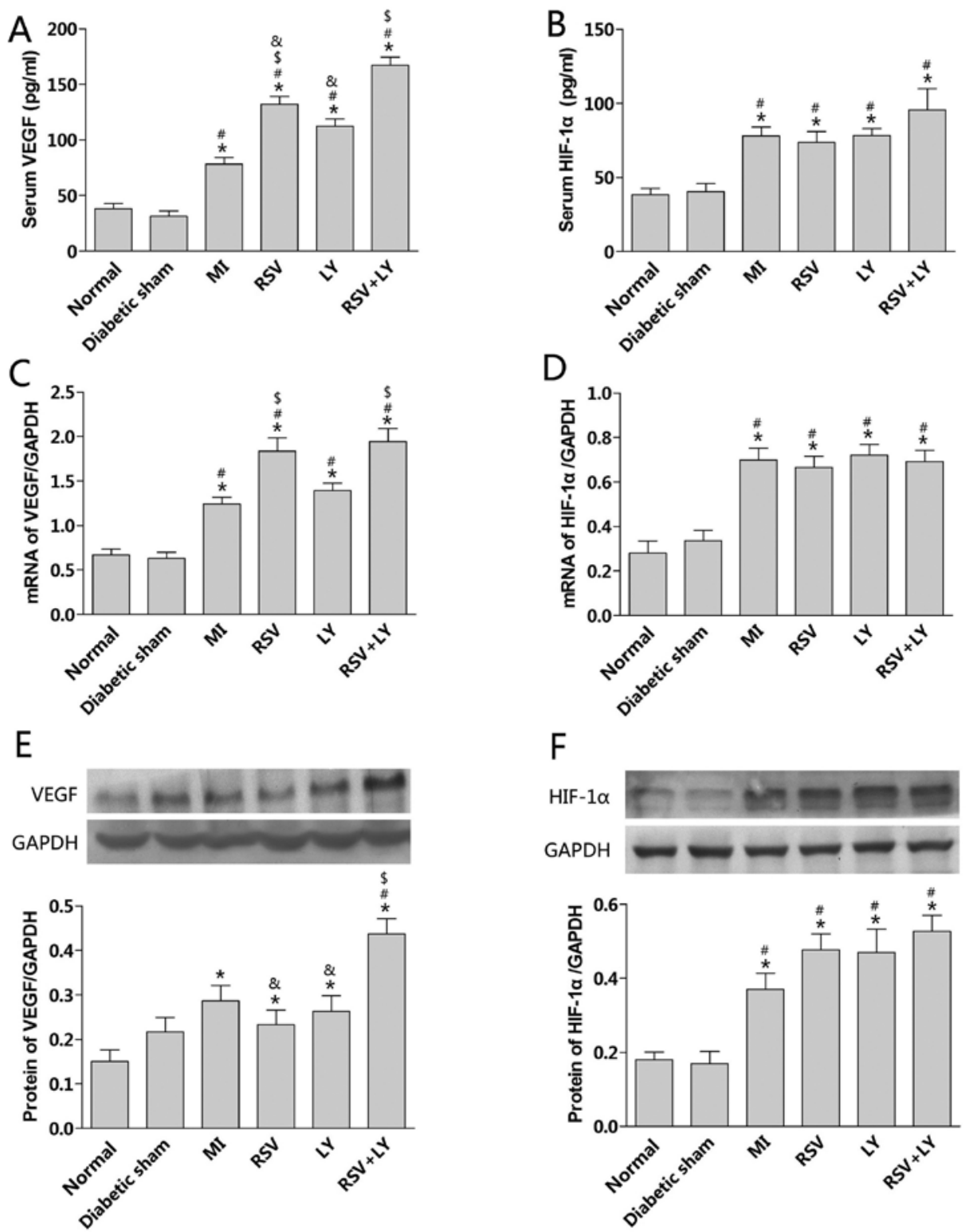

Figure 5. Protein levels of (A) VEGF and (B) hypoxia-inducible factor (HIF)-1 $\alpha$ in plasma were measured by ELISA. mRNA expression levels of (C) VEGF and (D) HIF-1 $\alpha$ in pre-infarct zone were measured by RT-qPCR. Protein expression levels of (E) VEGF and (F) HIF-1 $\alpha$ in the pre-infarct zone were measured by western blot analysis. Data are expressed as the means $\pm \mathrm{SD} ; \mathrm{n}=8-10$ rats per group. ${ }^{*} \mathrm{P}<0.05$ vs. normal group; ${ }^{*} \mathrm{P}<0.05$ vs. diabetic sham group; ${ }^{\$} \mathrm{P}<0.05$ vs. MI group; ${ }^{\text {P }}<0.05$ vs. RSV+LY group. VEGF, vascular endothelial growth factor; RSV, rosuvastatin; LY, LY333531; MI, myocardial infarction.

MI also increased the serum HIF-1 $\alpha$ protein levels and the myocardial HIF- $1 \alpha$ protein and mRNA levels $(\mathrm{P}<0.05$; Fig. 5B-F). The HIF-1 $\alpha$ levels were not significantly affected by single or combination treatment.

Effect of combination treatment with rosuvastatin and LY333531 on Akt and eNOS phosphorylation in rats. MI on its own did not induce Akt or eNOS phosphorylation (Fig. 6). Although there was a numerical increase in Akt phosphorylation following treatment with either rosuvastatin or LY333531 alone, the change did not reach statistical significance $(\mathrm{P}>0.05$; Fig. 6A), while treatment with rosuvastatin + LY333531 significantly increased Akt phosphorylation $(\mathrm{P}<0.05)$. Monotherapy with either rosuvastatin or LY333531 significantly increased eNOS phosphorylation $(\mathrm{P}<0.05$; Fig. 6B), and this increase was even more pronounced following treatment with rosuvastatin + LY333531 $(\mathrm{P}<0.05)$.

\section{Discussion}

The present study demonstrated that treatment with rosuvastatin or LY333531 reversed the high glucose-induced impairment of HUVEC tube formation and migration. These effects on HUVECs were further enhanced when the cells were treated with both rosuvastatin and LY333531. Using a rat diabetic model, we found that co-treatment with rosuvastatin and LY3333531, but not treatment with either agent alone, improved the cardiac function of the rats with MI. Oure results further revealed that co-treatment with rosuvastatin and LY333531 had a greater effect on capillary density in the peri-infarct zone 

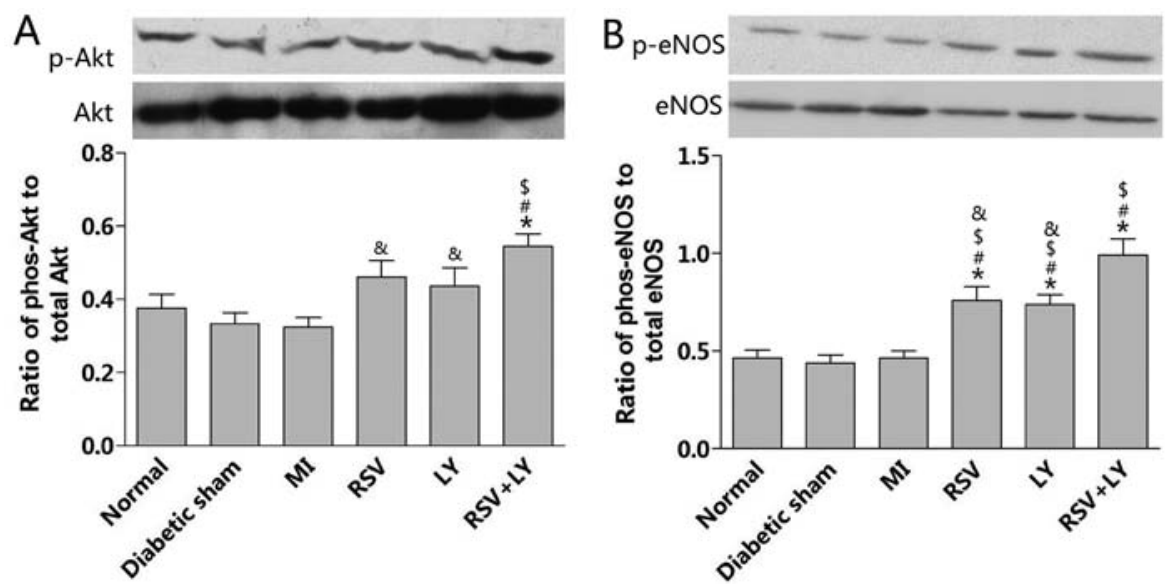

Figure 6. Representative western blots showing (A) phosphorylated Akt over total Akt protein expression and (B) phosphorylated eNOS over total endothelial nitric oxide synthase (eNOS) protein expression. Data are expressed as the means $\pm \mathrm{SD} ; \mathrm{n}=8-10$ rats per group. ${ }^{*} \mathrm{P}<0.05$ vs. normal group; ${ }^{*} \mathrm{P}<0.05$ vs. diabetic sham group; ${ }^{\$} \mathrm{P}<0.05$ vs. MI group; ${ }^{\circledR} \mathrm{P}<0.05$ vs. RSV + LY group. RSV, rosuvastatin; LY, LY333531; MI, myocardial infarction.

than treatment with either agent alone. This effect was mediated by the upregulation of the VEGF-dependent Akt/eNOS signaling pathway. Taken together, and to the best of our knoweledge, the results of the present study provide the first evidence of improved cardiac function following combination treatment with rosuvastatin and LY333531, as compared to treatment with either agent alone in a rat model of MI under diabetic conditions. In addition combination treatment had a pro-angiogenesis effect.

The coronary collateral circulation is a critical mechanism of adaptation of the heart to prevent cardiac dysfunction caused by ischemic insults $(29,30)$. The mechanisms underlying the development of coronary collaterals are largely unknown, and the role of myocardial ischemia as an inhibitory factor is unclear $(31,32)$. The degree of collateral coronary development varies greatly among patients with myocardial ischemia (33). A growing body of evidence suggests that diabetic patients experience accelerated atherosclerosis and, as shown angiographically and in autopsy studies, they also have an impaired ability to form coronary collaterals in response to myocardial ischemia $(33,34)$. In addition, in vitro studies have demonstrated decreased tree-like tubular network formation by HUVECs cultured under high glucose conditions (35). Different concepts have been proposed to explain the impaired angiogenic response under diabetic conditions (4). One of the commonly accepted mechanisms includes the decreased cardiac expression of VEGF and its receptors in diabetic states (9).

Statins have been suggested to exert cardioprotective benefits through mechanisms independent of lipid-lowering effects, including anti-inflammatory effects, improvement of endothelial function and NO synthesis. The angiogenic effects of statins remain controversial (36). Zaitone and Abo-Gresha (37) demonstrated that rosuvastatin promoted angiogenesis in diabetic rats with MI. The present study demonstrated that rosuvastatin significantly increased tube-like network formation and the migration of HUVECs cultured in high-glucose medium, whereas the formation of coronary collaterals was not further enhanced by rosuvastatin following the induction of MI in rats with diabetes. However, an increased VEGF expression was observed in the rosuvastatin- treated rats, indicating that factors other than decreased VEGF levels in the diabetic myocardium are involved in the impaired development of coronary collaterals. Hyperglycemia has been reported to induce the activation of $\mathrm{PKC} \beta 2$, which has been associated with diabetic complications, as treatment with the PKC 32 -specific inhibitor, LY333531, has been shown to be effective in diabetic myocardial hypertrophy, retinopathy and nephropathy $(21,38,39)$. Furthermore, it has been established that the hyperglycemia-induced activation of PKC $\beta 2$ inhibits the insulin-mediated Akt-dependent regulation of eNOS in obesity-associated insulin resistance (15). Consistently, our previous study confirmed that the inhibition of PKC $\beta 2$ by LY333531 not only increased the phosphorylation levels of Akt and eNOS, but also improved coronary collateral development in response to $\mathrm{MI}$ in diabetic rats, indicating that the activation of PKC $\beta 2$ may be involved in the downregulation of the Akt/eNOS angiogenic pathway under diabetic conditions (40). Indeed, in the present study, we observed that treatment with LY333531 alone increased the Akt-dependent eNOS activation without increasing VEGF expression. However, it has been demonstrated that the LY333531-associated inhibition of PKC $\beta 2$ enhances the myocardial ischemia-triggered increase in VEGF expression levels, leading to the improvement of impaired angiogenesis in diabetic rats (41).

Most importantly, the present study suggests that combination treatment with rosuvastatin and LY333531 is more effective in promoting the revovery of cardiac function than treatment with rosuvastatin or LY333531 alone. The extent of angiogenesis measured by CD31 staining also indicated that the combination treatment exerted more pronounced angiogenic effects compared to the other treatment groups. This is consistent with the findings regarding the changes in VEGF protein levels in the combination treatment group. Additionally, the extent of Akt and eNOS phosphorylation was more pronounced in the combination group than in the groups treated with rosuvastatin or LY333531 alone. Therefore, enhanced neovascularization in the infarcted myocardium induced by combination treatment with rosuvastatin and LY333531 seems to ameliorate dysfunctional ventricular remodeling. However, it has also been shown that treatment 
with both statins and LY333531 alone decreases the expression of TGF- $\beta 1$ with a concomitant decrease in myocardial fibrosis $(42,43)$. In the present study, we cannot exclude the possibility that the decreased extent of myocardial fibrosis by statins and LY333531 also contributed to the improvement of cardiac function. In particular, we did not observe a more pronounced decrease in serum TGF- $\beta 1$ protein levels in the group treated with the combination of both agents as compared to the groups treated with either agent alone.

In conclusion, to the best of our knowledge, the results of the present study suggest, for the first time, that treatment with rosuvastatin in combination with LY333531 is more beneficial compared to treatment with either statins or LY333531 alone during an MI event under diabetic conditions. The beneficial effects are likely attributed to the increased numbers of coronary collaterals.

\section{Acknowledgements}

The present study was supported a grant from the Science and Technology Commission of Shanghai Municipality to D.H. (no. 14ZR1432000).

\section{References}

1. Nordlie MA, Wold LE and Kloner RA: Genetic contributors toward increased risk for ischemic heart disease. J Mol Cell Cardiol 39: 667-679, 2005.

2. Habib GB, Heibig J, Forman SA, Brown BG, Roberts R, Terrin ML, et al: Influence of coronary collateral vessels on myocardial infarct size in humans: results of phase I Thrombolysis in Myocardial Infarction (TIMI) trial: the TIMI Investigators. Circulation 83: 739-746, 1991.

3. Samuel SM, Thirunavukkarasu M, Penumathsa SV, Koneru S, Zhan L, Maulik G, et al: Thioredoxin-1 gene therapy enhances angiogenic signaling and reduces ventricular remodeling in infarcted myocardium of diabetic rats. Circulation 121: 1244-1255, 2010.

4. Boodhwani M, Sodha NR, Mieno S, Xu SH, Feng J, Ramlawi B, et al: Functional, cellular, and molecular characterization of the angiogenic response to chronic myocardial ischemia in diabetes. Circulation 116 (11 Suppl): 131-137, 2007.

5. Martin A, Komada MR and Sane DC: Abnormal angiogenesis in diabetes mellitus. Med Res Rev 23: 117-145, 2003.

6. Shweiki D, Itin A, Soffer D and Keshet E: Vascular endothelial growth factor induced by hypoxia may mediate hypoxia-initiated angiogenesis. Nature 359: 843-845, 1992.

7. Hammes HP, Lin J, Bretzel RG, Brownlee M and Breier G: Upregulation of the vascular endothelial growth factor/vascular endothelial growth factor receptor system in experimental background diabetic retinopathy of the rat. Diabetes 47: 401-406, 1998.

8. Cooper ME, Vranes D, Youssef S, Stacker SA, Cox AJ, Rizkalla B, et al: Increased renal expression of vascular endothelial growth factor (VEGF) and its receptor VEGFR-2 in experimental diabetes. Diabetes 48: 2229-2239, 1999.

9. Chou E, Suzuma I, Way KJ, Opland D, Clermont AC, Naruse K, et al: Decreased cardiac expression of vascular endothelial growth factor and its receptors in insulin-resistant and diabetic States: a possible explanation for impaired collateral formation in cardiac tissue. Circulation 105: 373-379, 2002.

10. Mellor $\mathrm{H}$ and Parker PJ: The extended protein kinase $\mathrm{C}$ superfamily. Biochem J 332: 281-292, 1998.

11. Geraldes P and King GL: Activation of protein kinase C isoforms and its impact on diabetic complications. Circ Res 106: 1319-1331, 2010.

12. Inoguchi T, Battan R, Handler E, Sportsman JR, Heath W and King GL: Preferential elevation of protein kinase C isoform II and diacylglycerol levels in the aorta and heart of diabetic rats: differential reversibility to glycemic control by islet cell transplantation. Proc Natl Acad Sci USA 89: 11059-11063, 1992.

13. Sheetz MJ and King GL: Molecular understanding of hyperglycemia's adverse effects for diabetic complications. JAMA 288 2579-2588, 2002.
14. Wei L, Yin Z, Yuan Y, Hwang A, Lee A, Sun D, et al: A PKC-beta inhibitor treatment reverses cardiac microvascular barrier dysfunction in diabetic rats. Microvasc Res 80: 158-165, 2010.

15. Naruse K, Rask-Madsen C, Takahara N, Ha SW, Suzuma K, Way KJ, et al: Activation of vascular protein kinase C-beta inhibits Akt-dependent endothelial nitric oxide synthase function in obesity-associated insulin resistance. Diabetes 55: 691-698, 2006

16. He $\mathrm{Z}$ and King GL: Protein kinase $\mathrm{C}$ beta isoform inhibitors: a new treatment for diabetic cardiovascular diseases. Circulation 110: 7-9, 2004.

17. Sata M, Nishimatsu H, Osuga J, Tanaka K, Ishizaka N, Ishibashi $\mathrm{S}$, et al: Statins augment collateral growth in response to ischemia but they do not promote cancer and atherosclerosis. Hypertension 43: 1214-1220, 2004.

18. Erbs S, Beck EB, Linke A, Adams V, Gielen S, Kränkel N, et al: High-dose rosuvastatin in chronic heart failure promotes vasculogenesis, corrects endothelial function, and improves cardiac remodeling--results from a randomized, double-blind, and placebo-controlled study. Int J Cardiol 146: 56-63, 2011.

19. Siddiqui AJ, Gustafsson T, Fischer H, Widegren U, Hao X, Mansson-Broberg A, et al: Simvastatin enhances myocardial angiogenesis induced by vascular endothelial growth factor gene transfer. J Mol Cell Cardiol 37: 1235-1244, 2004.

20. Miura S, Matsuo Y and Saku K: Transactivation of KDR/Flk-1 by the $\mathrm{B} 2$ receptor induces tube formation in human coronary endothelial cells. Hypertension 41: 1118-1123, 2003.

21. Nakamura S, Chikaraishi Y, Tsuruma K, Shimazawa M, Hara H, et al: Ruboxistaurin, a PKCbeta inhibitor, inhibits retinal neovascularization via suppression of phosphorylation of ERK1/2 and Akt. Exp Eye Res 90: 137-145, 2010.

22. Shi F, Wang YC, Zhao TZ, Zhang S, Du TY, Yang CB, et al: Effects of simulated microgravity on human umbilical vein endothelial cell angiogenesis and role of the PI3K-Akt-eNOS signal pathway. PLoS One 7: e40365, 2012.

23. Huang FY, Mei WL, Li YN, Tan GH, Dai HF, Guo JL, et al: Toxicarioside A inhibits tumor growth and angiogenesis: involvement of TGF- $\beta /$ endoglin signaling. PLoS One 7: e50351, 2012.

24. Xin P, Zhu W, Li J, Ma S, Wang L, Liu M, et al: Combined local ischemic postconditioning and remote perconditioning recapitulate cardioprotective effects of local ischemic preconditioning. Am J Physiol Heart Circ Physiol 298: H1819-H1831, 2010.

25. Derumeaux G, Mulder P, Richard V, Chagraoui A, Nafeh C, Bauer F, et al: Tissue Doppler imaging differentiates physiological from pathological pressure-overload left ventricular hypertrophy in rats. Circulation 105: 1602-1608, 2002.

26. Tang JM, Wang JN, Zhang L, Zheng F, Yang JY, Kong X, et al: VEGF/SDF-1 promotes cardiac stem cell mobilization and myocardial repair in the infarcted heart. Cardiovasc Res 91: 402-411, 2011.

27. Xie J, Lu W, Gu R, Dai Q, Zong B, Ling L, et al: The impairment of ILK related angiogenesis involved in cardiac maladaptation after infarction. PLoS One 6: e24115, 2011.

28. Yu T, Zhu W, Gu B, Li S, Wang F, Liu M, et al: Simvastatin attenuates sympathetic hyperinnervation to prevent atrialfibrillation during the postmyocardial infarction remodeling process. J Appl Physiol 113: 1937-1944, 2012.

29. Chilian WM, Penn MS, Pung YF, Dong F, Mayorga M, Ohanyan V, et al: Coronary collateral growth - back to the future. J Mol Cell Cardiol 52: 905-911, 2012.

30. Hoole SP, White PA, Read PA, Heck PM, West NE and O'Sullivan $\mathrm{M}$, et al: Coronary collaterals provide a constant scaffold effect on the left ventricle and limit ischemic left ventricular dysfunction in humans. J Appl Physiol 112: 1403-1409, 2012.

31. Fujita M, Ikemoto M, Kishishita M, Otani H, Nohara R, Tanaka T, et al: Elevated basic fibroblast growth factor in pericardial fluid of patients with unstable angina. Circulation 94: 610-613, 1996.

32. Chilian WM, Mass HJ, Williams SE, Layne SM, Smith EE, Scheel KW, et al: Microvascular occlusions promote coronary collateral growth. Am J Physiol 258: H1103-H1111, 1990.

33. Abaci A, Oguzhan A, Kahraman S, Eryol NK, Unal S, Arinc $\mathrm{H}$, et al: Effect of diabetes mellitus on formation of coronary collateral vessels. Circulation 99: 2239-2242, 1999.

34. Yarom R, Zirkin H, Stammler G and Rose AG: Human coronary microvessels in diabetes and ischaemia. Morphometric study of autopsy material. J Pathol 166: 265-270, 1992. 
35. Dubois S, Madec AM, Mesnier A, Armanet M, Chikh K, Berney T, et al: Glucose inhibits angiogenesis of isolated human pancreatic islets. J Mol Endocrinol 45: 99-105, 2010.

36. Elewa HF, El-Remessy AB, Somanath PR and Fagan SC: Diverse effects of statins on angiogenesis: new therapeutic avenues. Pharmacotherapy 30: 169-176, 2010.

37. Zaitone SA and Abo-Gresha NM: Rosuvastatin promotes angiogenesis and reverses isoproterenol-induced acute myocardial infarction in rats: role of iNOS and VEGF. Eur J Pharmacol 691: 134-142, 2012.

38. Liu Y, Lei S, Gao X, Mao X, Wang T, Wong GT, et al: PKC $\beta$ inhibition with ruboxistaurin reduces oxidative stress and attenuates left ventricular hypertrophy and dysfunction in rats with streptozotocin-induced diabetes.Clin Sci (Lond) 122: 161-173, 2012.

39. Kelly DJ, Zhang Y, Hepper C, Gow RM, Jaworski K, Kemp $\mathrm{BE}$, et al: Protein kinase $\mathrm{C}$ beta inhibition attenuates the progression of experimental diabetic nephropathy in the presence of continued hypertension. Diabetes 52: 512-518, 2003.
40. Wang F, Huang D, Zhu W, Li S, Yan M, Wei M, et al: Selective inhibition of PKC $\beta 2$ preserves cardiac function after myocardial infarction and is associated with improved angiogenesis of ischemic myocardium in diabetic rats. Int $\mathrm{J}$ Mol Med 32: 1037-1046, 2013.

41. Ikeda A, Matsushita S and Sakakibara Y: Inhibition of protein kinase $C \beta$ ameliorates impaired angiogenesis in type I diabetic mice complicating myocardial infarction. Circ J 76: 943-949, 2012.

42. Ma YX, Li WH and Xie Q: Rosuvastatin inhibits TGF-betal expression and alleviates myocardial fibrosis in diabetic rats. Pharmazie 68: 355-358, 2013.

43. Palaniyandi SS, Ferreira JC, Brum PC and Mochly-Rosen D: PKC $\beta I I$ inhibition attenuates myocardial infarction induced heart failure and is associated with a reduction of fibrosis and pro-inflammatory responses. J Cell Mol Med 15: 1769-1777, 2011. 